\title{
A Method for Extracting Street Trees from Mobile LiDAR Point Clouds
}

\author{
Guowei Yue, Rufei Liu*, Heng Zhang and Maolun Zhou \\ Geomatics College, Shandong University of Science and Technology, Qingdao, Shandong, 266590, P.R. China
}

\begin{abstract}
This paper presents a method to extract street trees from laser scanning point clouds based on segmentation and growth of three dimensional (3d) grids. We firstly build the $3 \mathrm{~d}$ virtual grid of scanning area point clouds. Then, the distribution characteristic of point cloud is analyzed in a grid cell (density, elevation etc.). Finally, the point clouds of tree trunk and tree crown are extracted through grid segmentation and growth by a bottom-to-top way. In order to verify the validity of this method, we used the designed method to analysis the real collection of LiDAR point cloud data.
\end{abstract}

Keywords: Distribution characteristic, mobile laser scanning, point clouds extraction, segmentation and growth, threedimensional grid.

\section{INTRODUCTION}

Trees are an important part of urban ecosystem, there has a difficulty was how to survey space tree growth circumstance quickly. Mobile laser scanning technology as a kind of the measurement system was low cost, high precision and high efficiency, it provides a new technical means for obtain high resolution $3 \mathrm{~d}$ spatial information quickly and efficiently. Because of vast point cloud data of the measurement system, a hot spot of current research methodology from large point cloud to extract different features such as roads, buildings, trees.

In view of the trees point cloud data segmentation and feature extraction, some experts and scholars carried on the related research from different Angle. The main method has the following: (1) The method of feature space clustering [1, 2], This method uses point cloud spatial distribution of the local geometry for different features such as buildings, ground, trees are classified recognition and extraction by training samples; (2) The method of point cloud feature image analysis [3, 4], This method produce the characteristics of the cloud images and make full use of the threshold segmentation of image processing, this method extracting the boundary of the target buildings or trees; (3) The method of layer grid point density [5], This method will laser spot of the tree layer projection to the regular grid and computing the point density grid step by step, the laser spot of individual trees were extract; (4) The method of geometric characteristics [6, 7], this method adopts the top-down search strategies, it through the collection of parabolic surface fitting characteristics of the canopy, the canopy as the initiate to search the whole tree plant trees by a bottom-to-top way.

The method adopt different technical route for the classified point cloud data, but there were some disadvantages. (1) The method of feature space clustering training is difficult in the sample, especially for large scale scene was classification time-consuming and low efficiency; (2) The entire point cloud onto the plane XOY by the method of point cloud feature image analysis, this method showed the distance on the plane and elevation difference through features image, it reduced the point cloud data resolution, at the same time it has a difficult in selecting various parameters generate characteristics of the image; (3)The method of layer grid point density due to only considering the density of some information, and without considering spatial distribution characteristics of point cloud characteristics (such as elevation, the projection area, etc.), this method is limited for low interference feature on the ground floor around the trunk of the removal capacity; (4) The method of geometric characteristics find the boundary curve due to the need by curve fitting canopy, this method only extract a larger plant trees, it doesn't apply to cross crown an overlap. The three-dimensional point cloud was disposed by those methods, but it is large, inefficient. It doesn't suitable for large-scale scene point cloud processing. If we adopt the method of regular grid for static, it will reduce the resolution of the original data, and the limited scope of application.

According to the above problem, we proposed a method for extraction street tree quickly of face laser scan point cloud data. The method was based on a three-dimensional virtual grid index, it adopted segmentation and growth method to extract point cloud, and through the analysis of the spatial distribution of grid internal point cloud characteristics factor, we adopts a bottom-up strategy to extract the trees.

\section{ANALYSIS OF CHARACTERISTICS OF STRUC- TURE OF TREES}

Trees are one of the main landscape cities. Generally, it lined up in the road on both sides; there has a wide variety of trees, and large difference of morphological characteristics. According to the laws of the growth of tree and the external manifestation, the tree structure characterization factors mainly include tree height, diameter and canopy size, etc. As shown in Fig. (1). 


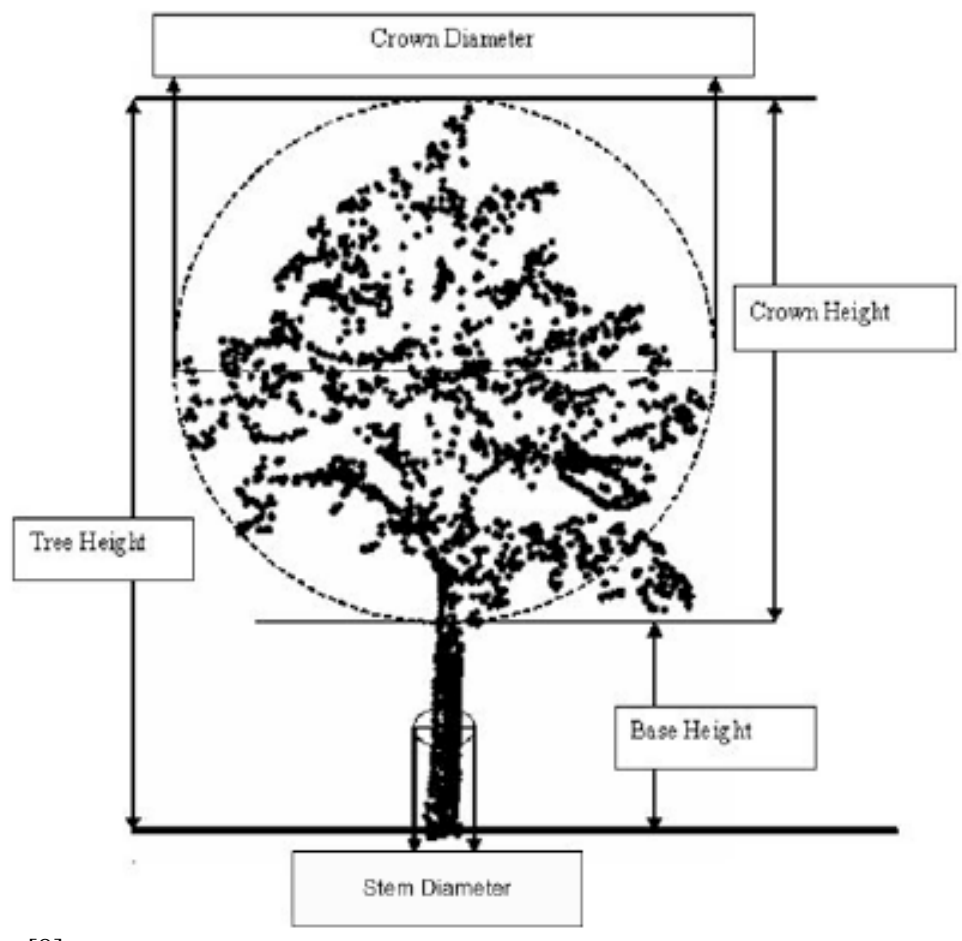

Fig. (1). Model structure of a tree [8].

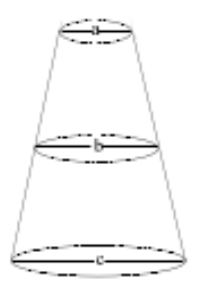

(a)

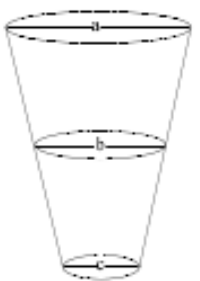

(b)

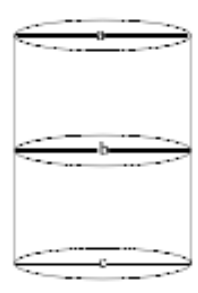

(c)

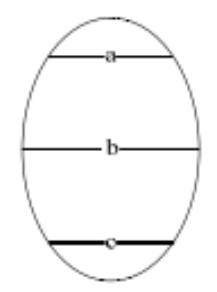

(d)

Fig. (2). Four forms of tree crown [8].

Vehicle scanning system can linear scanning along the direction of the road, each scan line corresponds to a road profile, when it scanning the tree and starting from the root of the tree to the trunk and the crown until the top of the tree, it takes the following two characteristics.

(1) The scan point cloud in the trunk section performance planar point clouds, it as the trunk surface point, Which are distributed into the shape of the general cylindrical, the point cloud density is equality, Low noise feature points is found around the bottom of the trunk, the same section of tree species and the same size.

The canopy due to the laser penetrating of in between leaves. Crown Point cloud performance the shape of $3 \mathrm{~d}$. The horizontal cross-section were took from the top, middle and bottom of crown, we are assuming diameters of a, b, c, It is not difficult to find the shape of the crown manifests four forms: If $\mathrm{a}<\mathrm{b}<\mathrm{c}$, it present the conical shape of the structure presented (Fig. 2a), a $>$ b $>$ c, it present the inverted cone structures (Fig. $\mathbf{2 b}$ ), $\mathrm{a} \approx \mathrm{b} \approx \mathrm{c}$, it present when rendering cylindrical configuration (Fig. 2c), $a<b$ and $b>c$, it present the rendering ellipsoid structure (Fig. 2d).

Fig. (3) is a street tree vehicle's point cloud data acquired by the Vehicle-mounted mobile laser scanning system. The

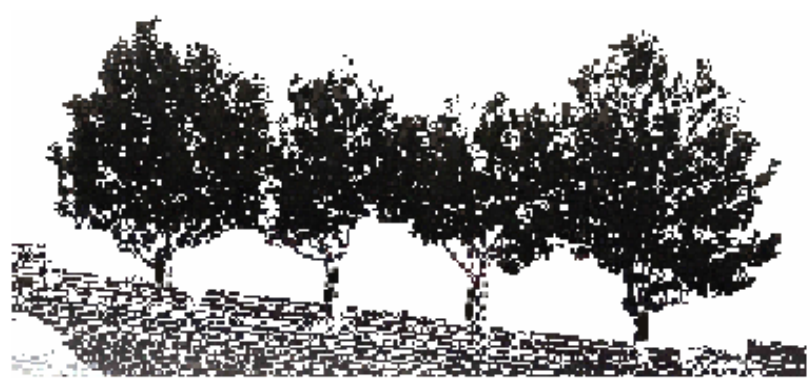

Fig. (3). Point clouds of street trees.

rules can be seen that the distribution of street tree trunk regular and the diameter of the same size. In Fig. (2), the c and $\mathrm{d}$ of the tree crown shape is most common. Due to the smaller spacing of street trees, the canopies of adjacent two trees were overlap.

\section{THE METHOD OF EXTRACTION TREES}

In this paper, based on the space distribution of trees in laser point cloud, a 3D virtual grid index is proposed [8-10]. Grids division and growth are processed through the analysis of the spatial distribution of grid internal point cloud characteristic factor. We can obtain the collection of a single layer 


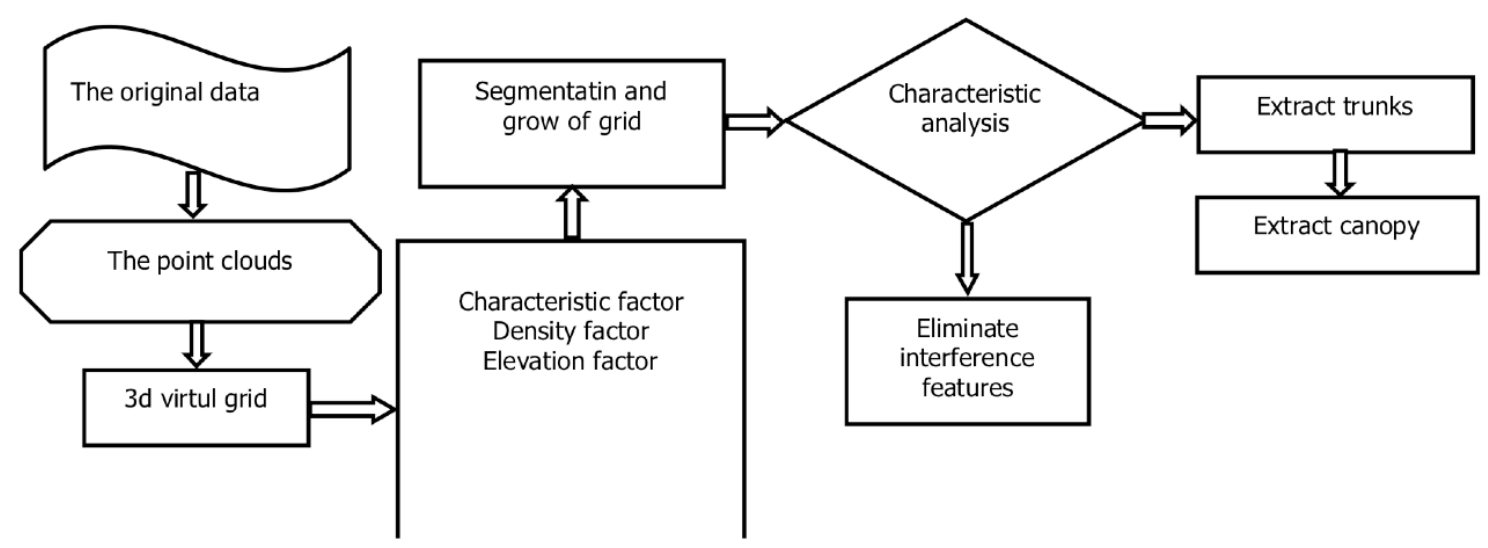

Fig. (4). Flowchart of the method for extracting street trees froth point clouds.

by growth to determine whether the trunk or crown. A bottom-up search strategy is used to achieve extraction of whole grain street trees. The overall technical process of the method is shown in Fig. (4).

\subsection{3d Virtual Grid Index}

The $3 \mathrm{~d}$ virtual grid diagram is shown in Fig. (5), the black point as the discrete laser sweep pastry cloud. The virtual grids were constituted by many layers of equal to the grid (cube), the high aspect was set according to the actual circumstance of scanning the area. The each laser point of point cloud data will accord the $3 \mathrm{~d}$ coordinate distribution, which will be divided into the appropriate grid cell. Because of each grid cell in virtual grid has a corresponding index, at the same time the rules of $3 \mathrm{~d}$ virtual grid was established will retain the original information of point cloud data. In this paper, we set up the index by the grid unit line number, column number and layer number, represent a grid cell by $\mathrm{G}(\mathrm{i}, \mathrm{j}, \mathrm{k})$.

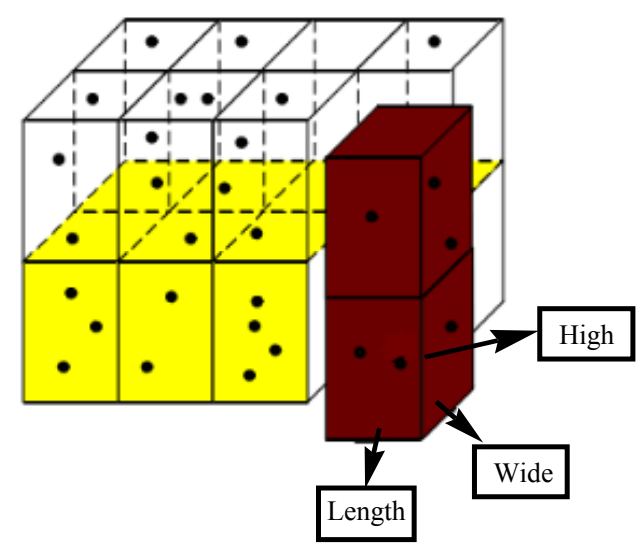

Fig. (5). Graph of 3D virtual grid.

\subsection{Characteristics of the Factor Analysis}

Due to the different features of $3 \mathrm{~d}$ shape is different, so they in the $3 \mathrm{~d}$ space density distribution and the height distribution of the laser spot is different. The point cloud data actually was get by the analysis of actual, we found that trees noise point is mainly divided into tree trunks noise point and tree canopy noise point, the former is mainly low vegetation near the trees, artificial ground objects, pedestrians and so on, its height is less than the general trunk; The latter is ver- tical light pole and billboards around trees in common, these artificial feature point cloud has bigger difference with the canopy. This paper is based on 3d virtual grid index and mainly analyzes the three dimensional laser spot in the grid spatial distribution density factor and elevation factor in the extraction of a bottom-up strategy.

\section{(1) The density factor}

The number of laser point represents the density factor. The number of laser points $(\mathrm{Dg})$ is counted in each grid cell, this value as the characteristic value of the density grid. The laser point value of size was related to the distribution of point cloud and the size of the grid.

The same trunk point cloud was distributed to the multilayer $3 \mathrm{~d}$ grid. In generally, because of the trunk has vertical distribution, the multi-layer grid has column number similarity, at the same time the grid has density threshold similarity. Based on this, according to the Car Scanner posture, the speed of the car and the distance from the edge of the road, we can make a deal of get point cloud data of actually and confirm the grid density threshold (Dt) of the single trunk. If the ground floor of the trunk grid contains low interference feature, it will be far greater than the point cloud density.

The tree canopy have large differences between different road street, when we determining the canopy grid density threshold, we using this statistics method of multiple tree canopy grid point density and gain point density modal interval [Dcmin, Dcmax] to determine canopy grid density values (Dc) of worshiping.

\section{(2) The elevation factor}

The $3 \mathrm{~d}$ grid cell as the basic unit of classification to obtain laser spot height values the maximum and the minimum in each grid cell and calculate the difference value between two values, with the difference value $(\mathrm{Hg})$ as the elevation characteristic value of three dimensional grid. The difference value of size and the distribution of point cloud were related to the size of the grid. According to the continuity of street trees in three-dimensional space, the trunk and canopy height factor are approximate to the height of the grid, in this paper, we set the threshold value of trunk and canopy as the elevation grid height. On the ground floor, the grid point that the elevation factor is less than the height within the grid as the ground clutter near around the canopy or other interference noise points. 


\subsection{Grid Gradual Segmentation and Growth Method}

We building $3 \mathrm{~d}$ virtual grid of point cloud and statistics the elevation difference and density of each grid point, we used the $\mathrm{D}$ as grid density factor and the $\mathrm{H}$ as grid elevation factor, according to the characters of street trees growth and the characteristics of the on-board scanning, the grid point may be determined as the laser spot on the trunk if it meet conditions (1), the grid point may be determined as the laser spot on the crown if it meet conditions (2).

$\left\{\begin{array}{l}D>D_{1} \\ H>H_{g}\end{array}\right.$.......(1)and $\left\{\begin{array}{l}D>D_{2} \\ H>H_{g}\end{array}\right.$......(2)

Ideally, we can get the trunk by search the grid cell that satisfies the above conditions, and extract the laser spot. But in the actual cases, the grid size is not necessarily meeting the requirements. We need the grid constantly progressive segmentation and growth.

(1) Make sure the virtual target grid

This paper put forward Trees extraction method by a bottom-up search method (as shown in Fig. 7), so the first things was make sure the trunk network in the ground layer grid. Find a mesh feature satisfies the formula (1) grid $\mathrm{G}(\mathrm{i}, \mathrm{j}, 1)$, We make it up vertical search and judge the characteristics of the top of the grid $\mathrm{G}(\mathrm{i}, \mathrm{j}, 2)$ in the second layer, if the grid $G(i, j, 2)$ also satisfy formula (1), then put $G(i, j, 2)$ labeled trunk on the ground floor grid.

\section{(2) Grid segmentation}

In the grid of ground floor, the trunk grid density contain low interference features was large, but the height of the interference ground feature less the grid height. In order to reduce the influence of the low interference terrain around the trunk, we performed gradual segmentation for the virtual three-dimensional grid and analyzed the characteristic of the grid after segmentation to further screened the interference figures.

The process of $3 \mathrm{~d}$ grid gradual segmentation: in the plane XOY, we performed gradual segmentation for length and width of $3 \mathrm{~d}$ mesh bottom. The mesh size is reduced to half of the original in the $\mathrm{X}$-axis and $\mathrm{Y}$-axis directions simultaneously. The $3 \mathrm{~d}$ grid mesh height remained unchanged. With the gradual increasing in the number, the number of $3 \mathrm{~d}$ grid of exponential grows exponentially. At the first time we suppose division the number of $3 \mathrm{~d}$ grid was $\mathrm{N}$, the number of $3 \mathrm{~d}$ mesh into $(22 \mathrm{k} * \mathrm{~N})$ by $\mathrm{k}$ gradual segmentation, as shown in Fig. (6).
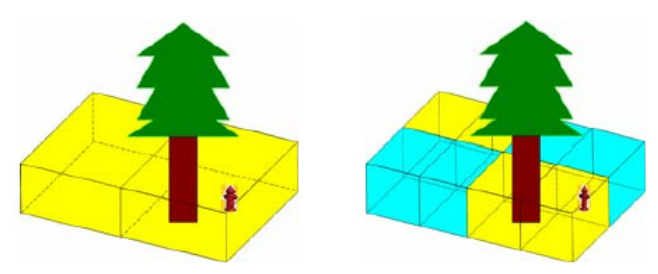

Fig. (6). Gradual segmentation of 3D virtual grid.

When the trunk floor grid $G(i, j, 1)$ not only contains the laser on the trunk, but also includes low noise Point cloud noise, such as some weeds, the density factor of $G(i, j, 1)$ would be significantly larger than the density factor of the second-tier trunk grid $\mathrm{G}(\mathrm{i}, \mathrm{j}, 2)$. In such a case, we needs to split grids into smaller progressively, until the point on the trunk and the noises surrounding the tree trunk were classified in different grid (as shown in Fig. 4). Grid size depends on the distance of the trunk and its interfering objects. According to the sampling results, the grid size is generally 10 cm level.

\section{(3) Growth of the grid}

(1) According to measurement result of the trees, the trunk diameter is generally $30-50 \mathrm{~cm}$. When the gradual segmentation of the grid size is reduced to $20 \mathrm{~cm}$ or even less, we need to make trunk grid gradual growth by a local neighborhood search methods. If the eight-neighbor mesh feature satisfy formulas (1) in the trunk grid $G(i, j, 1)$ of the ground floor, the neighborhood grids will be grown into tree trunks grid. In process of the grid growth, the grid containing only low objects, due to their density or height characteristic properties doesn't meet the trunk characteristic, it will be deleted.

(2) If trunk height is $\mathrm{Ht}$ and grid height is $\mathrm{Hg}$, the layer number of the trunk grid is $\mathrm{Hg} / \mathrm{Ht}$. The difference value between trunk grid line number and column number is close. On a single-layer grid, single tree trunk grid number is usually less than four. When there are trunk grid layer that twolayer are met trunk conditions, it belongs to the trunk. After it find a trunk floor grid collection, and then it will go into the second layer by the bottom-up search way and look for ground floor grid which similar rank exists. If it satisfies formulas (1), it will be joined to the trunk grid. It will go into the third layer and find upward trunk in turn.

(3) Because the street trees is continue in $3 \mathrm{~d}$, the tree crown points were distributed in multiple adjacent grid in the horizontal direction from the highest level trunk grid, the trees trunk in vertical and horizontal directions will start grow from the highest grid layer. If the layer number of the highest grid belonged to trunk grid is $\mathrm{k}$, the tree crown grid will grow at the $\mathrm{k}+1$ layer. Using neighborhood search methods in XOY plane, the 8 grid belonging to the outskirts of the collection of $k+1$ layer trunk grid, which satisfy the formula (2), if the grid satisfied the conditions that grow into tree crown grid collection.

For the adjacent trees, there have tree crown overlapping conditions. Because tree crown point clouds generally have duplication along the road direction and there is not overlapping in the vertical direction on the road, it will be constrained by using the grid range in the vertical direction on the road when the crown grid growth. In other words, when the tree crown growth of the road direction reaches such a scope will stop. Because the adjacent A and B tree crowns are without overlapping with the vertical direction on the road, this tree crown diameters can be calculated in this direction that is $a$ and $b$. Using center line of two tree trunks as axis to make two cylinders respectively, whose diameter is a and $\mathrm{b}$, laser point of two cylinder overlap are assigned evenly to the tree A and B.

(4) Tree crown is N times as big as the trunk grid areas (generally greater than 4). When the tree crown eightneighbor grid of all grids in the grid collection does not satisfy the formula (2), or stop searching when search to the 


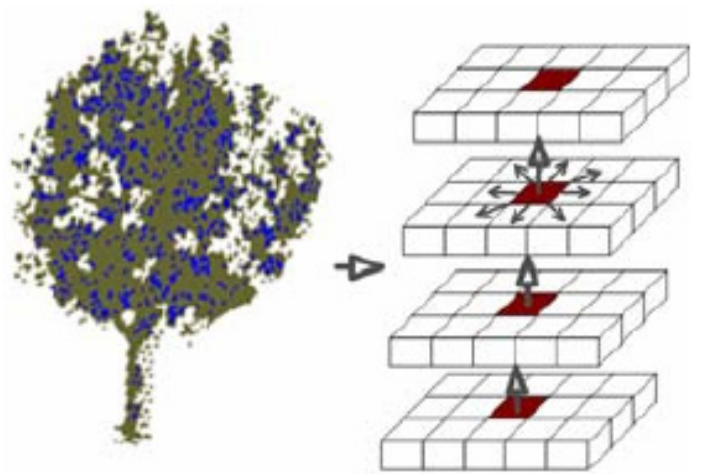

Fig. (7). Bottom-up search method for street trees.

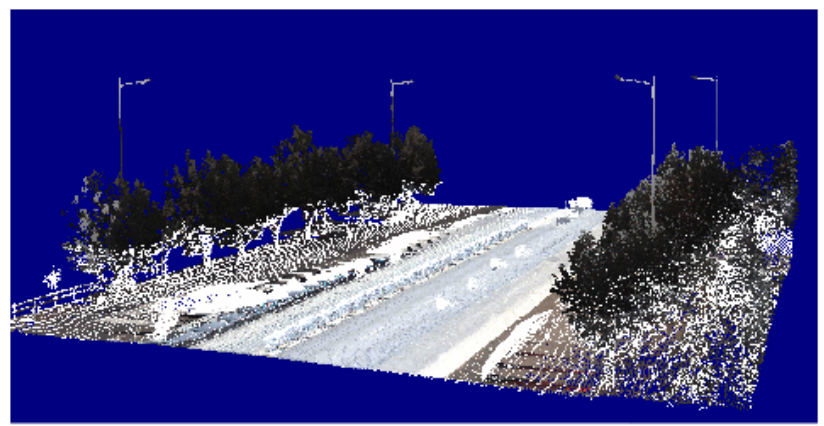

Fig. (8). Experimental data of point clouds.

grid border, at the same time grid shape of tree crown regional distribution will be judged. As shown in Fig. (2), if it is one of four forms will be marked output. It goes into last layer to find the whole crown and will be marked and output.

\section{TESTING AND ANALYSIS}

\subsection{Test Data}

This experiment adopt the laser scanning data of city blocks and landscape trees on both sides of the street, which were collected by the vehicular $3 \mathrm{~d}$ movement measurement system V-Surs of the Qingdao mountain mobile measuring company, as shown in Fig. (8). The region has a total of 1203881 laser spot. We can observe from the figure, there are two rows street trees that grow neatly in the besides the road, and there are overlapping areas between the lush green canopy. There are some other features such as telephone poles in the middle of the trees and weed low distractions under the tree.

\subsection{Tree Extraction Results}

As shown in Fig. (8), we used the extraction method to process the point cloud data. Street tree can be screened out on both sides of road by gradual segmentation and growth to the $3 \mathrm{~d}$ virtual grid and analyze the grid density factor and the elevation factor, as shown in Fig. (9).

(1) The results of the extraction individual tree: We excluded vehicle jitter and pedestrian occlusion on collecting data by on-board scanner, according to the actual collect data, when the established grid size is $0.2 \mathrm{~m} * 0.2 \mathrm{~m}$, the height of the grid $(\mathrm{Hg})$ is $1 \mathrm{~m}$, we set the grid density factor threshold of Dt and Dc are 20 laser point, the individual tree without cover was extracted by the method in Fig. (10).

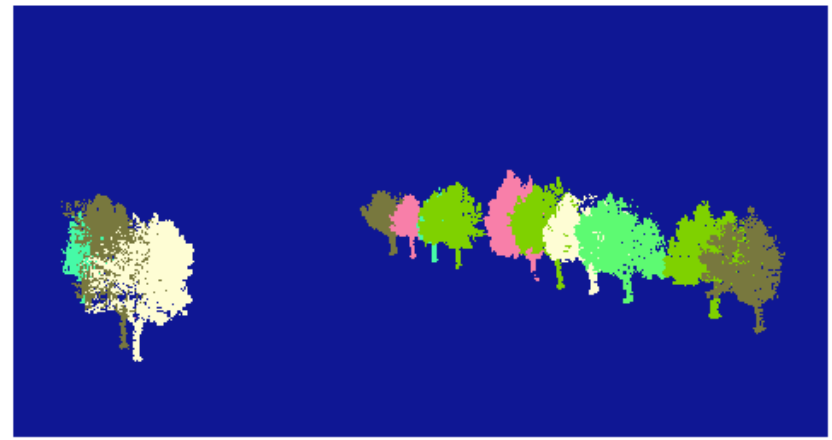

Fig. (9). Extraction result of street trees.

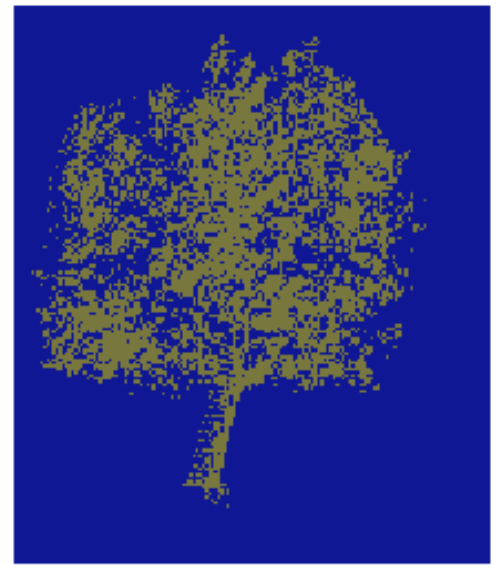

Fig. (10). Extraction result of individual street tree.

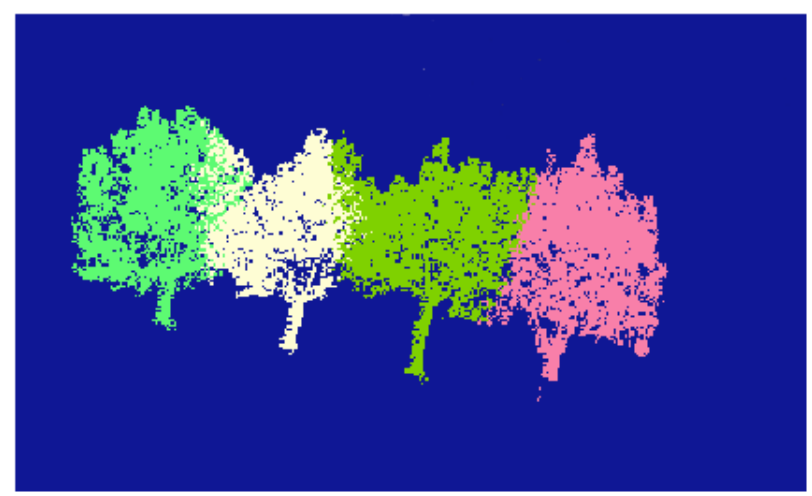

Fig. (11). Extraction result of street trees when tree crowns overlapping.

(2) As shown in Fig. (11), the proposed processing method was used to extraction the trees that the crown was extracted has overlap, when the established grid size is $0.2 \mathrm{~m}$ $* 0.2 \mathrm{~m}$, the height of the grid $(\mathrm{Hg})$ is $1 \mathrm{~m}$, we set the grid density factor threshold of Dt and Dc are 20 laser point, The point cloud data of overlapping part was distributed to the adjacent two trees evenly and applied colors to a drawing by different color, but due to some reasons such as Angle of view, we can't be fully from the renderings.

(3) The low interference around the trunk feature can be eliminated, the low interference in the ground floor grid can be eliminated effectively by the grid gradual segmentation, when the established grid size is $0.4 \mathrm{~m} * 0.4 \mathrm{~m}$, the height of the grid $\mathrm{Hg}$ is $1 \mathrm{~m}$, we set the grid density factor threshold of Dt and Dc are 40 laser point, the information of street trees 


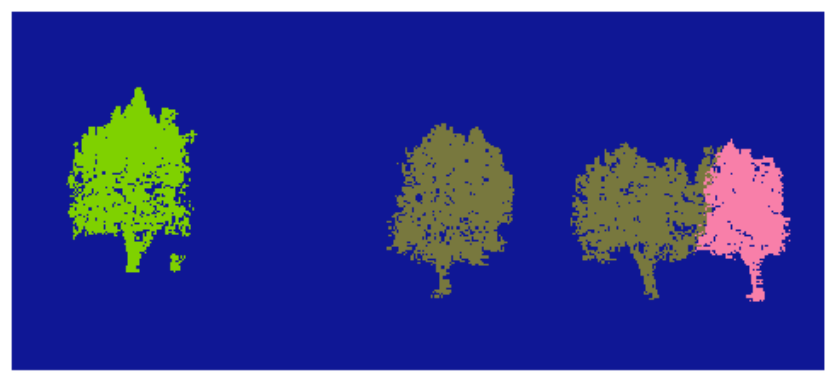

Fig. (12). Extraction result of street trees when noise objects existing.

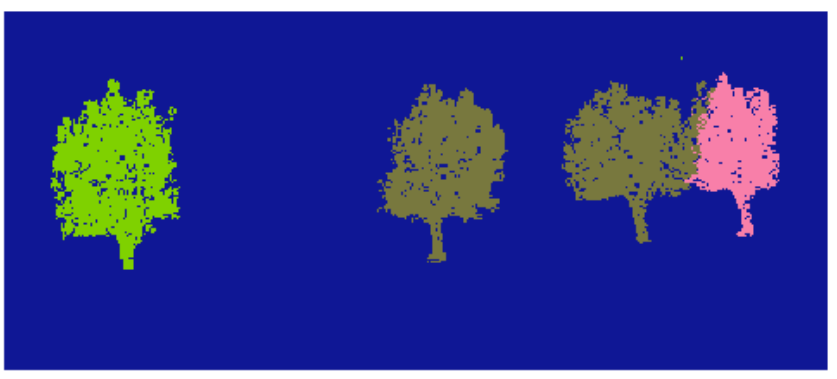

Fig. (13). Extraction result of street trees when excluding noise object.

can be extracted by analysis the test data. At the moment, the debris cannot be removed effectively on the ground. As shown in Fig. (12), there has low interference features exist in the left side of the trunk. The grid was gradual spitted further. We set the grid size of $0.2 \mathrm{~m} * 0.2 \mathrm{~m}$, the height of the grid $\mathrm{Hg}$ is $1 \mathrm{~m}$, and set the grid density factor threshold of $\mathrm{Dt}$ and Dc are 20 laser point, the low interference feature can be eliminated, and the trees was extracted by bottom to top way. As shown in Fig. (13).

\section{CONCLUSION}

This article is based on $3 \mathrm{~d}$ virtual grid index and analysis the grid density factor and elevation factor for grid gradual segmentation and growth. The growth was completed, the canopy and trunk was judged by analysis the distribution characteristics of point cloud from grid layer. In the end, the whole tree was searched by bottom-up strategy. The real point cloud data of city blocks can be analyzed by this method that extracts the trees effectively. But the actual shape of the trees in the scene were complicated, this method still needs further optimization. Because the dense canopy cross or cover between the two or more trees, the laser spot of overlapping area will be classified into two trees. In follow-up studies, we will further optimize structure and efficiency of algorithm in order to improve this method.

\section{CONFLICT OF INTEREST}

The authors confirm that this article content has no conflict of interest.

\section{ACKNOWLEDGEMENTS}

This study is supported by National key scientific instrument and equipment development projects (2013YQ120343), National Key Technology Research and Development Program of the Ministry of Science and Technology of China (2012BAB16B01), Marine Research Special Funds for Public Welfare Projects (201305034-1), Special Project Fund of Taishan Scholars of Shandong Province, and A Project of Shandong Province Higher Educational Science and Technology Program (J13LH04). The authors thank anonymous reviewers for their constructive comments and suggestions.

\section{REFERENCES}

[1] J. F. Lalonde, N. Vandapel, and D. F. Huber, "Natural terrain classification using three-dimensional ladar data for ground robot mobility," Journal of Field Robotics, vol. 23, no. 10, pp. 839-861, 2006.

[2] D. Munoz, N. Vandapel, and M. Hebert, "On board contextual classification of 3-D point clouds with learned high-order markov random fields," IEEE International Conference on Robotics and Automation (ICRA*09), Tohoku University, Japan, 2009.

[3] B. Yang, Z. Wei, and Q. Li. "For vehicular laser scanning point cloud fast classification characteristics of point cloud image generation method," Surveying and Mapping, vol. 39, no. 5, pp. 540$545,2010$.

[4] Z. Wei, Z. Dong, and Q. Li. "On-board LiDAR point cloud building facade automatically extract the boundary," Journal of Wuhan University Information Science Edition, vol. 37, no. 11, pp. 13111315, 2012.

[5] B. Wu, B. Yu, and W. Yue, "A method of individual trees information extraction base on vehicular laser scanning point cloud data," Journal of East China Normal University(Natural Science edition), vol. 2, pp. 38-49, 2013.

[6] Yi. Lin, J. Hyyppa, and A. Jaakkola. "Three-level frame and RDschematic algorithm for automatic detection of individual trees from MLS point clouds," International Journal of Remote Sensing, vol. 33, no. 03, pp. 1701-1716, 2012.

[7] B. Tan, R. Zhong, and Q. Li, Vehicular, "Laser scanning data object classification method," Journal of Remote Sensing, vol. 16, no. 1, pp. 50-66, 2012.

[8] M. Rutzinger, A. K. Pratihast, S. Oude Elberink, and G. Vosselman, "Detection and modeling of $3 \mathrm{~d}$ trees from mobile laser scanning data. International Archives of Photogrammetry", Remote Sensing and Spatial Information Sciences, vol. 38, no. 05, pp. 520$525,2010$.

[9] L. Gong, Y. Zhang, and Z. Li. "A method of airborne LIDAR data filtering based on multi-scale virtual grid and Gradient threshold," Science of Surveying and Mapping, vol. 37, no. 1, pp. 101-103, 2012.

[10] H. Zhang, X. Jia, Y. Zhang, and G. Dong, "Airborne LIDAR data filtering based on virtual grid and improve the slope filtering," Journal of Surveying and Mapping Science and Technology, vol. 26, no. 3, pp. 224-227, 2009.

This is an open access article licensed under the terms of the Creative Commons Attribution Non-Commercial License (http://creativecommons.org/licenses/by-nc/3.0/) which permits unrestricted, non-commercial use, distribution and reproduction in any medium, provided the work is properly cited. 